\begin{tabular}{|c|l|}
\hline Title & Time dependent phase behavior of 5CB-DDA B-water microemul sions monitored by means of dielectric spectroscopy \\
\hline Author(s) & Chen, Zhen; Nozaki, Ryusuke \\
\hline Citation & $\begin{array}{l}\text { Physical Review E, 84(1),011401 } \\
\text { https://doi.org/10.1103/PhysRevE.84.011401 }\end{array}$ \\
\hline Issue Date & 2011-07 \\
\hline Doc URL & http://hdl.handle.net/2115/47004 \\
\hline Rights & ○2011 The A merican Physical Society \\
\hline Type & article \\
\hline File Information & PRE84_1_011401.pdf \\
\hline
\end{tabular}

Instructions for use 


\title{
Time-dependent phase behavior of 5CB-DDAB-water microemulsions monitored by means of dielectric spectroscopy
}

\author{
Zhen Chen and Ryusuke Nozaki* \\ Department of Physics, Faculty of Science, Hokkaido University, Sapporo 060-0810, Japan
}

(Received 4 March 2011; published 14 July 2011)

\begin{abstract}
The phase behavior of an inverse microemulsion composed of 4-cyano-4- $n$-pentylbiphenyl (5CB), didodecyl dimethyl ammonium bromide, and water was intensively studied recently, but controversy still remains on the existence of a "transparent nematic" (TN) phase. Previously, we studied the temperature-dependent dielectric behavior of this microemulsion [Z. Chen and R. Nozaki, J. Chem. Phys. 134, 034505 (2011)]. The results suggested that a superstructure composed of water droplets and confined 5CB molecules exists in the coexistence phase region, which may correspond to the notion of a TN phase. In this study, the time-dependent dielectric behaviors of this microemulsion in the isotropic, coexistence, and supercooled phase regions were investigated. In the coexistence and supercooled phase regions, the dielectric behaviors showed strong dependence on time, due to the hysteresis of phase transition and the upward diffusion of the water-droplet-rich isotropic phase. After the isotropic phase has been totally excluded out of the effective measurement area, the slow process was still observed in these two phase regions, which suggests that water droplets can stably exist in the liquid crystalline phase. A local microscopic phase evolution was observed in the coexistence phase region, which is similar to the temperature-induced phase evolution previously proposed by us. The dielectric behavior of the slow process during the phase evolution also suggests the existence of the superstructure. In addition, the mechanism of the slow process in different phase regions proposed in our previous study is also confirmed.
\end{abstract}

DOI: 10.1103/PhysRevE.84.011401

PACS number(s): 82.70.Uv, 77.22.Gm, 47.57.jb

\section{INTRODUCTION}

The dispersion of surfactant-coated water droplets in a nematic liquid crystal (LC) host has attracted great interest in recent years [1-11]. Because of the elastic distortion of the LC host by the dispersed droplets and the interdroplet interaction mediated by the elastic distortion, this kind of water-inLC (micro)emulsion often exhibits unusual properties and fascinating colloidal superstructures. These properties and superstructures are generally absent in conventional (micro)emulsions but important in technological applications and fundamental science. For example, Poulin et al. reported an inverse LC emulsion of micron-sized water droplets dispersed in nematic 4-cyano-4- $n$-pentylbiphenyl (5CB) [1-3], in which the chaining of water droplets induced by the orientational elasticity of the LC host was directly observed by optical microscope. This direct evidence then facilitates the theoretical understanding on topological defect and particle interaction in nematic LC [2].

As compared with LC emulsion, liquid crystal microemulsion (LCM), in which the water droplets are nanosized, is less understood. Because of the large difference in the droplet size of more than two orders of magnitude, the colloidal structures formed in LCMs are expected to be very different from those in LC emulsions $[6,11,12]$. A typical LCM composed of 5CB, water, and surfactant didodecyl dimethyl ammonium bromide (DDAB) has been intensively studied recently [6-8,10,11], but controversy still remains on its phase behavior. On the basis of the optical observation and differential scanning calorimetry, Yamamoto and Tanaka [6] proposed that on cooling, this LCM exhibits two phase transformations: first

*nozaki@ dielectrics.sci.hokudai.ac.jp from an isotropic phase to a phase called "transparent nematic" (TN) phase, and then to a coexistence of bulklike nematic phase and TN phase. The TN phase is thought macroscopically transparent but locally ordered on scales of several nanometers. However, the results of later studies $[7,8]$ on the same system contradict the notion of the TN phase. It was proposed that the nanosized water droplets act more as molecular contaminants and therefore this LCM actually undergoes a phase demixing due to the expulsion of water droplets by the orientational ordering.

In order to provide some information on the controversy, we previously studied the phase transition of the same LCM as a function of temperature by means of dielectric spectroscopy (DS) [13]. Despite the complexity of the phase composition of the system, it was found that the bulklike 5CB molecules in different states can be well distinguished by the nature of their corresponding relaxation behaviors. In addition to these bulklike relaxations, an additional relaxation located in the lower-frequency side of them was observed in all phase regions, which strongly depends on the phase transition and has a different relaxation mechanism in different phase region (for simplicity we will call this low-frequency relaxation uniformly as "slow process" hereinafter despite the phase region). The abnormal temperature dependence of the slow process after the isotropic-to-nematic phase transition suggested that there exists a superstructure composed of water droplets and confined 5CB molecules. This superstructure may correspond to the notion of the TN phase, although it remains in a shorter length scale.

In this study we offer some additional information, still from the dielectric point of view, on the phase behavior of 5CB-DDAB-water microemulsion. The phase transition of this system is accompanied by a macroscopic phase separation due to the effect of gravity $[8,10]$. The upward diffusion of the 
droplet-rich isotropic phase will lead to local growth of the nematic phase and meanwhile a diminishment of the isotropic phase at the bottom of the sample, which may locally induce a similar microscopic phase evolution to the temperatureinduced phase evolution [13]. It is thereby tempting to locally monitor the time-dependent phase behavior of this system at a certain temperature, which is expected to provide some alternative insight on the phase transition and phase separation of this LCM. Due to its substantial advantages including noninvasion, high sensitivity, and quick measurement [14], DS is also an ideal tool in real-time monitoring kinetic processes, such as polymerization [15,16], crystallization [17-19], and even chemical reaction [20]. We use DS to real-time monitor the time-dependent dielectric behaviors of the same LCM in the isotropic, coexistence, and supercooled phase regions in this study. It will be demonstrated that the phase composition of this LCM depends not only on temperature but also on time, and that the existence of the previously mentioned superstructure is further confirmed by the time-dependent dielectric behavior of this LCM in the coexistence phase region.

\section{EXPERIMENT AND METHODS}

\section{A. Materials and sample preparation}

The liquid crystal 4-cyano-4- $n$-pentylbiphenyl (5CB) was purchased from Sigma-Aldrich and used as received. The surfactant didodecyl-dimethyl-ammonium-bromide (DDAB) was purchased from Tokyo Chemical Industry (Japan) and used without further purification. Water was distilled and deionized.

The preparation procedure of the liquid crystal microemulsion was simply described in our previous paper [13], which is the same as used in other studies [6-10]. The weight fraction $\left(\phi_{m}\right)$ of the water droplet (coated by DDAB) in the sample is 0.15 (denoted as $0.15 \mathrm{LCM}$ hereinafter). We choose this sample because it has the greatest dielectric response in all phase regions as compared with other LCMs investigated in our previous study [13]. Prior to the dielectric measurement, the sample was subjected to ultrasonication at $60^{\circ} \mathrm{C}$ for $40 \mathrm{~min}$ to ensure homogeneity.

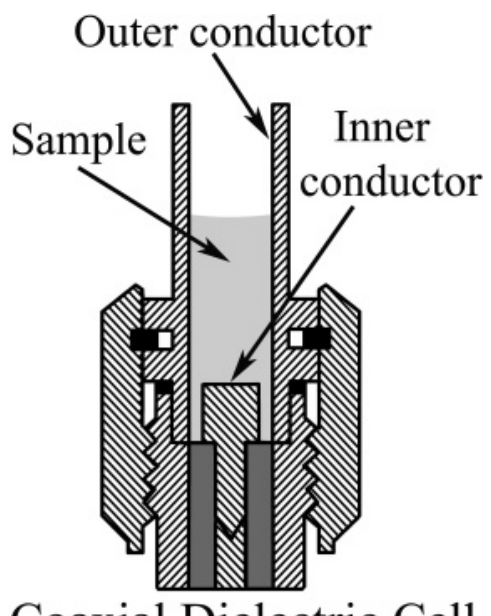

\section{Coaxial Dielectric Cell}

FIG. 1. Schematic illustration of coaxial dielectric sample cell.

\section{B. Dielectric measurement}

The complex dielectric permittivity $\left(\varepsilon^{*}=\varepsilon^{\prime}-i \varepsilon^{\prime \prime}\right.$, where $\varepsilon^{\prime}$ and $\varepsilon^{\prime \prime}$ are dielectric constant and dielectric loss, respectively) was measured by a low-frequency impedance analyzer (Agilent 4294A) in the frequency range from $40 \mathrm{~Hz}$ to $110 \mathrm{MHz}$. The values of the complex permittivity were obtained from reflection measurements with a coaxial sample cell located at the end of a coaxial line. The sample cell, schematically illustrated in Fig. 1, is composed of an outer conductor with an inner diameter of $3.5 \mathrm{~mm}$ and an
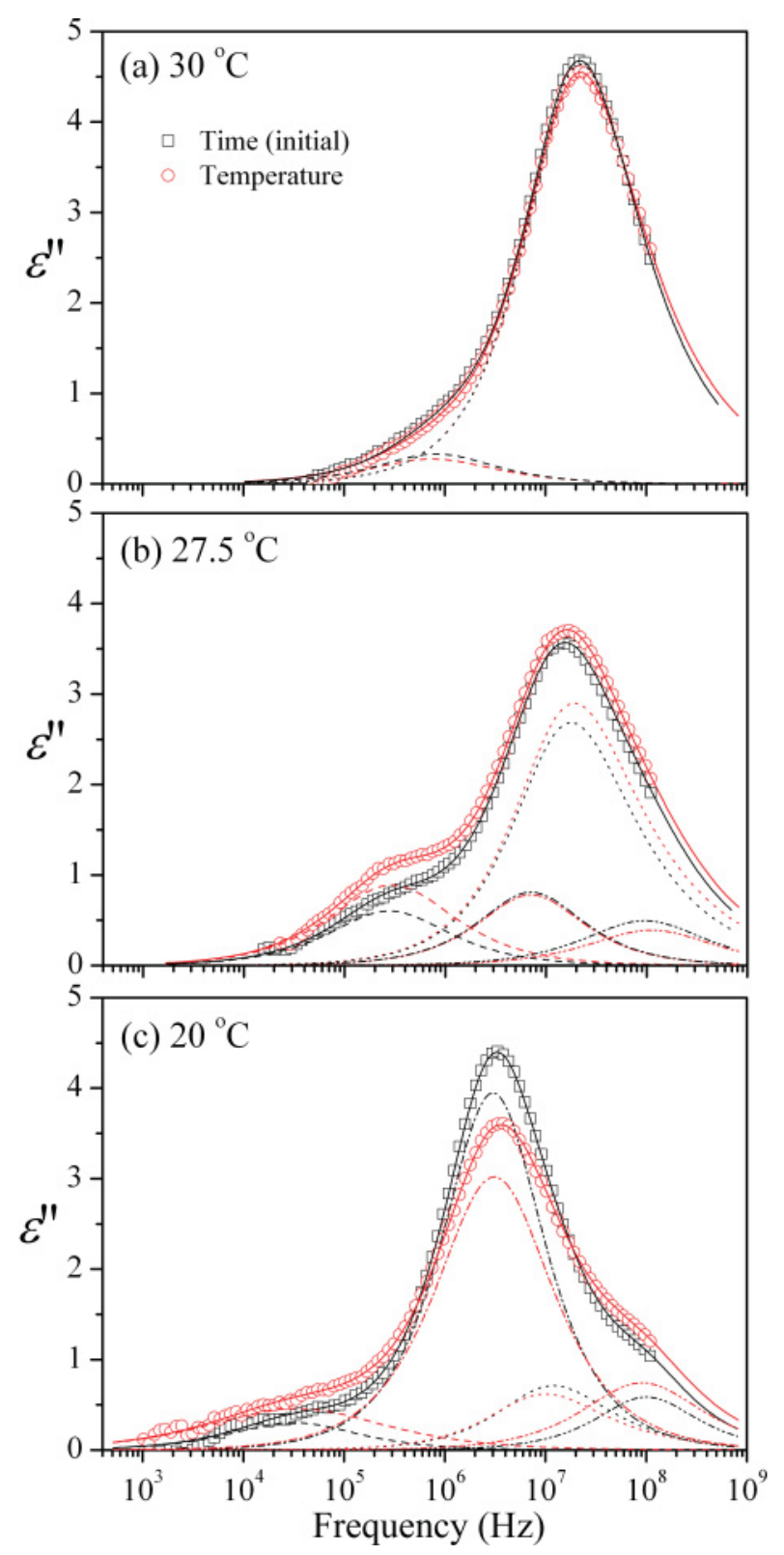

FIG. 2. (Color online) Frequency dependence of dielectric loss at different temperature of the $0.15 \mathrm{LCM}$ in the case of time dependence (initial state of this study) and in the case of temperature dependence (previous study). Solid line: the best fit; dashed line: slow process; dotted line: random reorientation of isotropic 5CB molecules; dashdot-dash line: rotation of $5 \mathrm{CB}$ molecule about the short axis; dashdot-dot line: librational motion. 
inner conductor (pin) with an outer diameter of $2 \mathrm{~mm}$. The lengths of the inner and outer conductor are 2 and $25 \mathrm{~mm}$, respectively. Only the bottom part of the sample that is located in the gap between the inner and outer conductors (effective measurement area) can be effectively measured.

The 0.15 LCM has two obvious phase transformations upon cooling [13]: an isotropic-to-nematic (IN) phase transition at $\sim 27.5^{\circ} \mathrm{C}$, and a nematic-to-supercooled nematic (NS) phase transition in the temperature range of $24^{\circ} \mathrm{C}-20^{\circ} \mathrm{C}$. Correspondingly, there are three phase regions called the isotropic region, coexistence region, and supercooled region, respectively. In the supercooled region, most $5 \mathrm{CB}$ molecules are in the supercooled nematic state, which coexist with water droplets and a small amount of 5CB molecules in the isotropic state. Therefore, we chose to perform the dielectric measurement as a function of time at $30^{\circ} \mathrm{C}, 27.5^{\circ} \mathrm{C}$, and $20^{\circ} \mathrm{C}$, respectively, corresponding to the three phase regions. For each temperature course, we used newly prepared 0.15 LCM and cooled it from $60{ }^{\circ} \mathrm{C}$ to the destination temperature, and then started the time-dependent measurement. Considering that the phase composition of LCM might be very sensitive to the cooling history, and that different phase composition will result in different dielectric behavior, we used the same measurement procedure as used in our previous study above the destination temperature, so as to keep the cooling history as consistent as possible. It should be pointed out, however, that it is impossible to make the cooling history exactly the same.

The temperature was controlled by using a compact ultralow-temperature chamber (MC-811, ESPEC Corp., Japan) with an accuracy of $\pm 0.1^{\circ} \mathrm{C}$. The duration of the dielectric measurement at each fixed temperature is $\sim 27.2 \mathrm{~h}$. We found that the dielectric behavior of the sample changes most intensively in the first hour or so; therefore the sample was measured about every $6 \mathrm{~min}$ in the first $72 \mathrm{~min}$ and then every $1 \mathrm{~h}$ in the following $26 \mathrm{~h}$. The dielectric behavior was automatically recorded by using VEE PRO 7.0 software (Agilent) at different time intervals.

\section{Determination of relaxation parameters}

The following fitting function is employed to fit the experimental complex permittivity spectra:

$$
\varepsilon^{*}(\omega)=\varepsilon_{\infty}+\sum_{j} F_{j}^{*}(\omega)+\frac{\sigma_{\mathrm{dc}}}{i \omega \varepsilon_{0}},
$$

where $\varepsilon_{\infty}$ is the high-frequency limit of the permittivity, $\varepsilon_{0}$ is the dielectric constant of vacuum, the term $F_{j}^{*}(\omega)$ accounts for the contribution of each dielectric relaxation, and the term $\sigma_{\mathrm{dc}} / i \omega \varepsilon_{0}$ accounts for the contribution of $\mathrm{dc}$ conductivity $\sigma_{\mathrm{dc}}$. For $F_{j}^{*}(\omega)$, we use the Havriliak-Negami function [21] or the Cole-Cole function [22], resting with the nature of each relaxation. The Havriliak-Negami function is given by

$$
F_{j}^{*}(\omega)=\frac{\Delta \varepsilon_{j}}{\left[1+(i \omega \tau)^{\beta}\right]^{\alpha}},
$$

where $\Delta \varepsilon_{j}$ is the dielectric strength, $\tau\left(=1 / 2 \pi f_{0}\right)$ is the relaxation time, $f_{0}$ is the characteristic frequency, and $\beta$ $(0<\beta \leqslant 1)$ and $\alpha(0<\alpha \leqslant 1)$ are the parameters describing the shape of the curve. When $\alpha=1$ and $0<\beta \leqslant 1$, Eq. (2) goes to the Cole-Cole function.

The mechanism of each relaxation observed in the 0.15 LCM in different phase regions has been discussed previously [13]. Accordingly, in the isotropic region, we use one ColeCole function for the slow process and one Havriliak-Negami function for the bulklike relaxation of isotropic 5CB that is
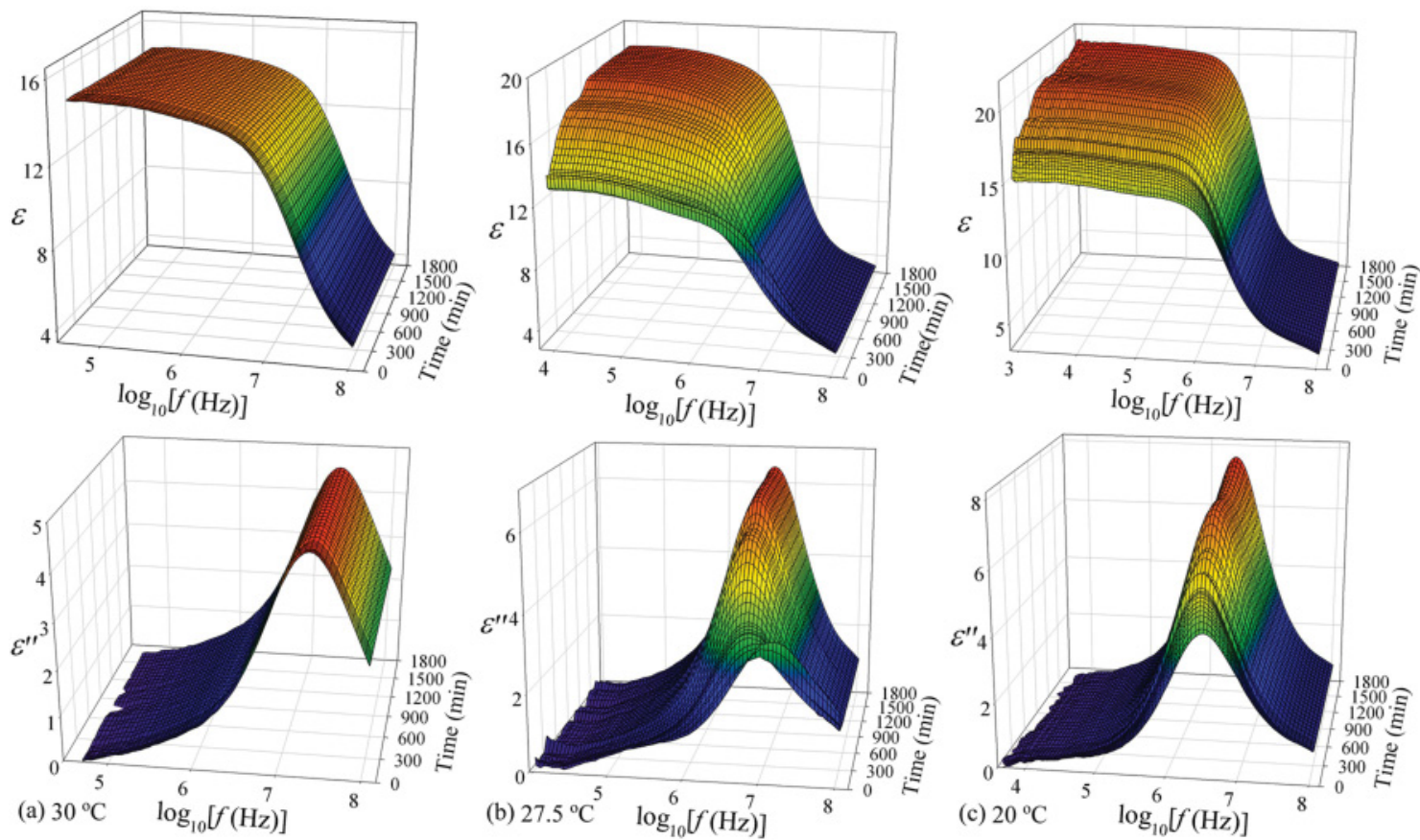

FIG. 3. (Color online) Three dimensional plots of the time dependence of relative permittivity spectra (top) and dielectric loss spectra (bottom) of the $0.15 \mathrm{LCM}$ at (a) $30^{\circ} \mathrm{C}$, (b) $27.5^{\circ} \mathrm{C}$, and (c) $20^{\circ} \mathrm{C}$. The contribution of the dc conductivity to the dielectric loss spectra has been subtracted. 
asymmetric. In the coexistence and supercooled regions, we use three Cole-Cole functions for the slow process, the rotation of 5CB molecules about their short axis, and the librational motion, respectively, and one Havriliak-Negami function for the relaxation of isotropic 5CB molecules. Figure 2 shows the dielectric loss spectra (after subtracting the dc conductivity) of the initial state of the $0.15 \mathrm{LCM}$ at (a) $30^{\circ} \mathrm{C}$, (b) $27.5^{\circ} \mathrm{C}$, and (c) $20^{\circ} \mathrm{C}$; the experimental data of the previous study (temperature dependence) at the same temperatures are also presented as comparison. The solid lines in Fig. 2 represent the best fits in line with Eq. (1) and the broken lines denote the contribution from different relaxations.

As can be seen in Fig. 2, the dielectric behaviors in the isotropic region [Fig. 2(a)] of both cases are nearly consistent with each other, but an obvious discrepancy exists when the sample is in the coexistence [Fig. 2(b)] and supercooled regions [Fig. 2(c)], and the longer the cooling history, the larger the discrepancy. Accordingly, we believe the discrepancy is mainly ascribed to the difference in the cooling history. Some other factors such as sample preparation and environmental effect may also be responsible for the discrepancy. Nevertheless, we stress that the characteristic relaxation times in both cases, within experimental error, are identical with each other for all relaxations. This suggests that the phase compositions of the samples in both cases are quite similar. In other words, if the factor of time is not taken into account and a similar cooling history is followed, the phase composition of LCM should be kept analogous.

\section{RESULTS AND DISCUSSION}

Figure 3 shows the three-dimensional (3D) plots of the relative permittivity and dielectric loss (after subtracting the dc conductivity) of the $0.15 \mathrm{LCM}$ as functions of frequency and time in the (a) isotropic region, (b) coexistence region, and (c) supercooled region. As can be seen, the dielectric behavior in the isotropic region barely changes with time, while in the coexistence and supercooled regions it changes obviously with time, especially at the early stage.

\section{A. Time-dependent dielectric behavior at $30^{\circ} \mathrm{C}$ (isotropic region)}

In the isotropic region, LCMs are like conventional waterin-oil microemulsions because 5CB molecules are disordered and behave like a normal organic solvent. Two dielectric relaxations can be observed: a slow process due to the retarded rotation of the $5 \mathrm{CB}$ molecules on the surface of the water droplets and a bulklike process due to the random reorientation of the bulk 5CB molecules [13].

Figure 4 shows the time dependence of the dielectric behavior of the $0.15 \mathrm{LCM}$ at $30^{\circ} \mathrm{C}$, where (a), (b), and (c) show the time dependence of the relaxation time $(\tau)$, the dielectric strength $(\Delta \varepsilon)$, and the dc conductivity $\left(\sigma_{\mathrm{dc}}\right)$, respectively. As can be seen, the dielectric behavior of bulklike relaxation changes barely with time, with constant $\tau$ and $\Delta \varepsilon$ through the whole duration, indicating that the isotropic 5CB molecules are very stable. For the slow process, its $\Delta \varepsilon$ keeps constant within the initial $300 \mathrm{~min}$ and then decreases with time. One cannot ascribe this decrease in $\Delta \varepsilon$ to experimental error, because $\Delta \varepsilon$ in the final state is $\sim 20 \%$ smaller than that in the initial state. Because the density of the surfactant-coated water droplet is smaller than that of bulk 5CB [8], this decrease should be a result of the upward diffusion of the water droplets. Considering that the experiment lasted a long period of time, a small amount of water may be evaporated, which may somewhat accelerate the upward diffusion of the water droplets. However, since the effective measurement area is far from the LCM-air interface (Fig. 1), the effect of the evaporation to the diffusion rate should be negligible as compared with that of the gravity.

It is interesting to find that $\sigma_{\mathrm{dc}}$ increases considerably in the initial $300 \mathrm{~min}$ and then levels off. Similar to that in normal water-in-oil microemulsions, the mechanism of conductance in the isotropic phase is believed mainly ascribed to the migration of charged water droplets in the electric field, namely, the charge fluctuation model $[23,24]$. Therefore, $\sigma_{\mathrm{dc}}$ is decided by

$$
\sigma_{\mathrm{dc}}=\frac{\varepsilon_{0} \varepsilon k_{B}}{2 \pi \eta r^{3}} T \phi_{v},
$$

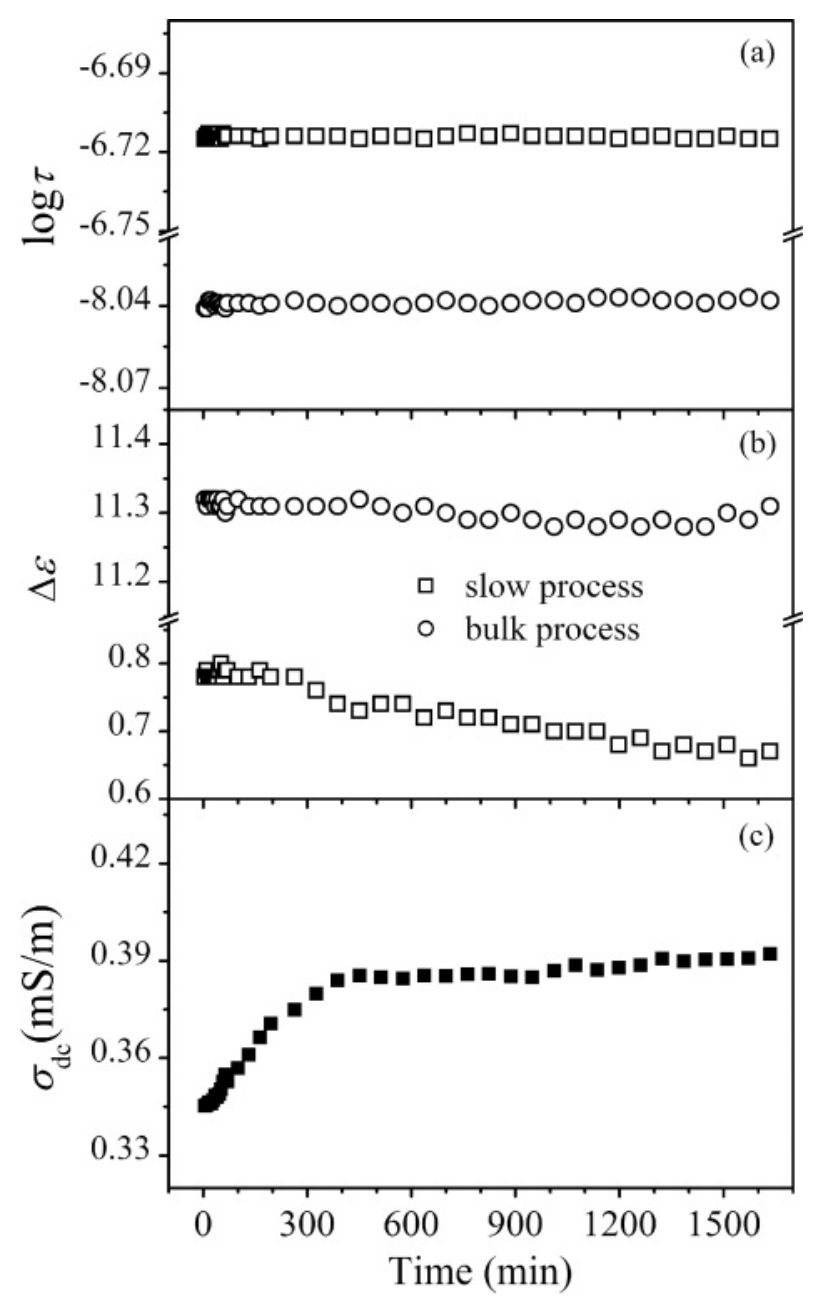

FIG. 4. Time dependence of (a) relaxation time, (b) dielectric strength, and (c) dc conductivity of slow process (open square) and bulklike process (open circle) of the $0.15 \mathrm{LCM}$ at $30^{\circ} \mathrm{C}$. 

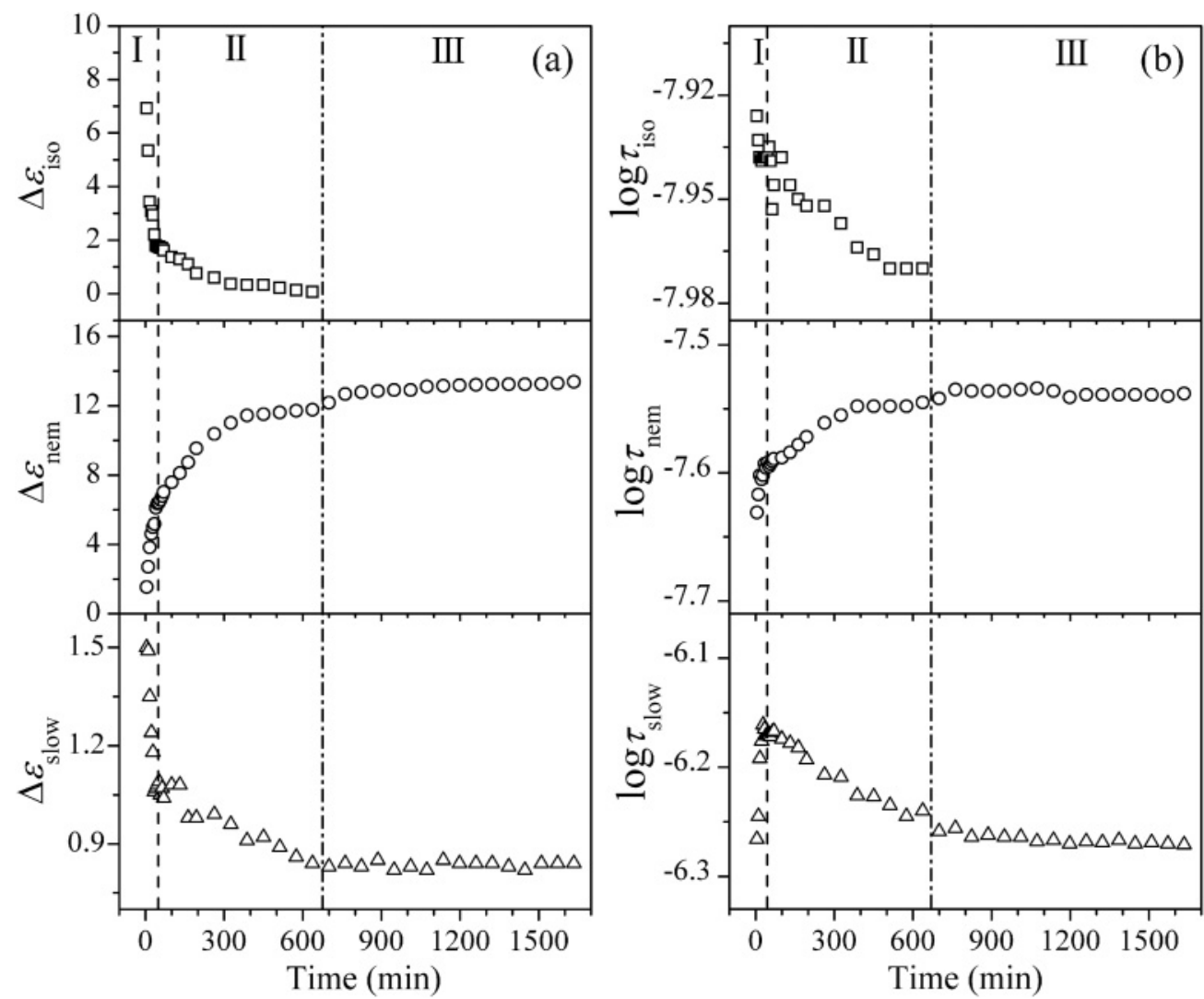

FIG. 5. Time dependence of (a) dielectric strength and (b) relaxation time of random reorientation of isotropic 5CB molecules (open square), rotation about short axis of nematic $5 \mathrm{CB}$ molecules (open circle), and slow process (open triangle) of the $0.15 \mathrm{LCM}$ at $27.5{ }^{\circ} \mathrm{C}$. The broken lines indicate the boundaries of different time regions.

where $\varepsilon$ and $\eta$ are the dielectric constant and viscosity of the solvent, respectively; $r$ and $\phi_{v}$ are the radius and the volume fraction of water droplets, respectively; $k_{B}$ is the Boltzmann constant; and $T$ is absolute temperature. Because $\phi_{v}$ cannot increase with time and $\eta$ stays nearly constant, the increasing $\sigma_{\mathrm{dc}}$ with time suggests that the fluctuation model alone cannot explain the dc conductance. Considering the relatively high $\phi_{v}$, the formation of short water droplet clusters due to droplet aggregation may play an important role in the conductance. In the initial $300 \mathrm{~min}$, since the phase composition of the system is very stable, the increase in $\sigma_{\mathrm{dc}}$ is most likely a result of the increasing probability of the formation of water droplet clusters as time elapses. After $300 \mathrm{~min}$, the much less intensive increase in $\sigma_{\mathrm{dc}}$ with time is possibly because the probability of the formation of water droplet clusters is largely decreased due to the upward diffusion of water droplets. The coalescence of water droplet is another possibility accounting for the increasing $\sigma_{\mathrm{dc}}$ with time, as observed in water-in-oil emulsions [25].

It is worth noting that the relaxation time of the slow process does not change with time, even after the water droplets start to diffuse upward. This suggests that the slow process in the isotropic phase is not much correlated with the state of water droplets, otherwise a relevant time dependence of $\tau$ to that of $\sigma_{\mathrm{dc}}$ should be observed. In other words, this process is not attributed to the dynamics of water droplets. It is therefore strongly confirmed that this process is attributed to the retarded rotation of $5 \mathrm{CB}$ molecules on the surface of the water droplets, whose relaxation time should be mainly a function of the viscosity of the surrounding $5 \mathrm{CB}$ medium.

\section{B. Time-dependent dielectric behavior at $27.5^{\circ} \mathrm{C}$ (coexistence region)}

After the IN phase transition, a phase demixing occurs and results in a coexistence of a denser droplet-poor nematic phase and a droplet-rich isotropic phase [6-10]. If the sample is kept at constant temperature for a long enough period of time, a macroscopic phase separation will occur due to the diffusion of the droplet-rich isotropic phase to the upper part of the container $[8,10]$. The dielectric behavior of LCM in the coexistence region is characterized by a slow process, which is believed due to the cooperative dynamics of 5CB molecules confined in a superstructure composed of water droplets, and bulklike relaxations arising from not only the nematic 5CB molecules but also the isotropic 5CB molecules [13]. With the upward diffusion of the droplet-rich isotropic phase, the phase composition of the sample in the effective measurement area varies with time and results in a time-dependent dielectric behavior as shown in Fig. 3(b).

Figure 5 shows the time dependence of (a) $\Delta \varepsilon$ and (b) $\tau$ of the $0.15 \mathrm{LCM}$ at $27.5^{\circ} \mathrm{C}$, where the subscript "iso," "nem," and "slow" represent the cases of isotropic, nematic, and confined $5 \mathrm{CB}$ molecules, respectively. It should be pointed out that, although there are two relaxations ascribed to nematic 5CB molecules - the rotation about the short axis and the librational 
motion-we use the former one to represent the dielectric behavior of nematic 5CB because it is much less vulnerable to fitting uncertainty [13]. As can be clearly seen, there exist three time regions labeled by the different time dependences of the relaxation parameters.

In time region I (within $\sim 60 \mathrm{~min}$ ), the dielectric behavior of all relaxations has the most intensive dependence on time. Because the relaxations under discussion are due to molecular dynamics, their dielectric strength can be described by the Kirkwood-Fröhlich equation [26,27]:

$$
\Delta \varepsilon=\frac{\varepsilon_{s}\left(\varepsilon_{\infty}+2\right)^{2}}{3\left(2 \varepsilon_{s}+\varepsilon_{\infty}\right)} \frac{g \mu_{0}^{2}}{9 \varepsilon_{0} k_{B} T} \frac{N}{V},
$$

where $\varepsilon_{s}$ is the static dielectric constant, $N / V$ is the number concentration of dipoles in a volume $V$, and $g$ is the Kirkwood correlation factor $\left(g=\mu_{\text {eff }}^{2} / \mu_{0}^{2}, \mu_{0}\right.$ and $\mu_{\text {eff }}$ are the mean square and effective dipole moment, respectively). Accordingly, $\Delta \varepsilon$ is a measure of the amount (concentration) of the $5 \mathrm{CB}$ molecules in the corresponding state: isotropic, nematic, or confined (in the superstructure) [13]. The remarkable decrease in $\Delta \varepsilon_{\text {iso }}$ and increase in $\Delta \varepsilon_{\text {nem }}$ indicate that the droplet-rich isotropic phase in the effective measurement area is quickly diminishing. There are two possibilities accounting for the quick diminishment: one is the upward diffusion of the isotropic phase, and the other is the hysteresis of the IN phase transition, that is, the transformation of the isotropic 5CB molecules to the nematic state persists for a period of time. The hysteresis of the IN phase transition is possibly due to the weakly first-order nature of the IN transition of this kind of system or due to some effect related to the configuration of the sample cell, e.g., the wetting and prewetting of the sample on the surface of the electrode. The self-diffusion coefficient of bulk 5CB molecules is estimated to be of the order of $10^{-10} \mathrm{~m}^{2} / \mathrm{s}$ [28]. Considering that the diffusion of the isotropic $5 \mathrm{CB}$ molecules in the LCM is influenced by the water droplets and the coexisting nematic phase, the effective self-diffusion coefficient of the isotropic 5CB molecules should be even smaller. This means it will take more than $6 \mathrm{~h}$ for them to diffuse a distance of $2 \mathrm{~mm}$ (the height of the inner conductor). However, $\Delta \varepsilon_{\text {iso }}$ is decreased by $\sim 75 \%$ during this short period of time, which means most isotropic phase has disappeared. Accordingly, the main reason should be the hysteresis of IN phase transition rather than the upward diffusion. Since most water droplets are thought to be present in the isotropic phase, some superstructures will be excluded out of the effective measurement area due to the phase transition, which gives rise to a large decrease of $\Delta \varepsilon_{\text {slow. }}$. On the other hand, the paranematic fluctuation increases with the increasing nematic phase $[8,11]$, which will cause the superstructure to become more compact and then decrease the confinement space of the $5 \mathrm{CB}$ molecules confined in the superstructure. This may be responsible for the obvious increase of $\tau_{\text {slow }}$ in this time region.

In time region II (60-700 $\mathrm{min}$ ), although much less intensive, the time dependence of $\Delta \varepsilon$ for bulklike relaxations is similar to that in time region I, which means the isotropic phase keeps diminishing while the nematic phase keeps growing. This should be mainly a result of the diffusion of the isotropic phase out of the effective measurement area. We notice that the magnitude of $\Delta \varepsilon_{\text {nem }}$ is doubled through this period of time. Such a large increase cannot be solely a result of the increase of the concentration of the nematic phase. We believe that the ordering parameter $(S)$ of the nematic phase is also considerably increased during this period of time, considering that a larger $S$ can give rise to a larger $\mu_{\text {eff }}$ and then a bigger $\Delta \varepsilon_{\text {nem }}$ [see Eq. (4)]. Figure 6 shows the time dependence of (a) the Cole-Cole parameter $\beta$ and (b) $\sigma_{\mathrm{dc}}$ of the sample at $27.5^{\circ} \mathrm{C}$. The Cole-Cole parameter is a measure of the distribution of relaxation times [29]. The closer the value of $\beta$ is to unity the narrower the distribution of relaxation times, which means the relaxing dipolar units are more uniform. From Fig. 6(a) we can find the value of $\beta_{\text {nem }}$ is very close to unity, indicating that the nematic phase is highly ordered. Although not markedly, $\beta_{\text {nem }}$ increases with time in both time regions I and II, which signifies that the nematic phase is more and more ordered, namely, $S$ is increasing with time. Meanwhile, from Fig. 5(b) we can see $\tau_{\text {nem }}$ is also increasing with time, which is another indication of the increase of $S$ with time.

Considering time regions I and II together, we find that $\Delta \varepsilon_{\text {iso }}$ is initially much bigger than $\Delta \varepsilon_{\text {nem }}$ and finally much smaller than $\Delta \varepsilon_{\text {nem }}$, suggesting that the sample is initially dominated by the isotropic phase but finally dominated by the nematic phase as time elapses. This situation is quite similar to the temperature-induced phase evolution in the coexistence region as proposed in our previous study [13]. Likewise, the sample should also undergo a phase evolution from a nematic-in-isotropic phase via a bicontinuous phase to an isotropic-in-nematic phase within $700 \mathrm{~min}$. In time region I, the sample is in the nematic-in-isotropic phase and then in the bicontinuous phase, where the isotropic phase should be

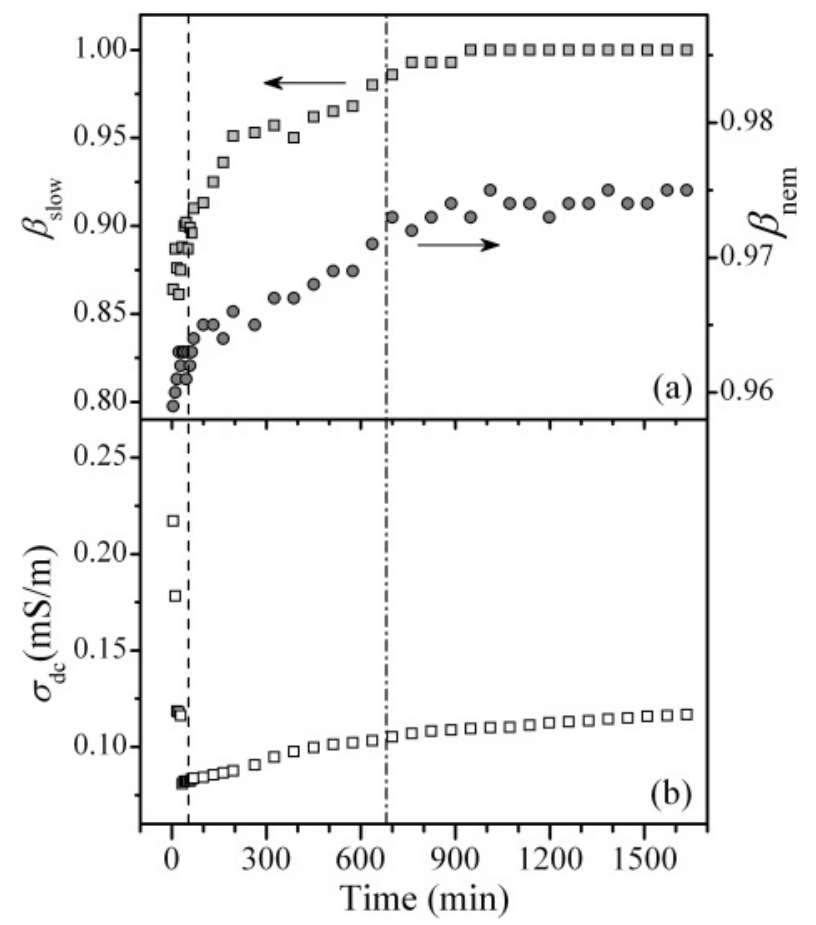

FIG. 6. Time dependence of the Cole-Cole parameter corresponding to (a) the slow process (filled square) and the relaxation due to short axis rotation of nematic 5CB molecules (filled circle) and (b) dc conductivity at $27.5^{\circ} \mathrm{C}$. The broken lines indicate the boundaries of different time regions. 
the dominant phase; while in time region II, the sample is in the isotropic-in-nematic phase dominated by the nematic phase. This scenario is supported by the time dependence of $\sigma_{\mathrm{dc}}$ [Fig. 6(b)]. In time region I, $\sigma_{\mathrm{dc}}$ is much larger than that in time region II and $\sigma_{\mathrm{dc}}$ has a similar time dependence to that of $\Delta \varepsilon_{\text {iso }}$. Considering that $\sigma_{\mathrm{dc}}$ in the isotropic phase is much larger than in the nematic phase [13], this result suggests that the isotropic phase is the dominant (continuous) phase in this time region. In time region II, however, $\sigma_{\mathrm{dc}}$ is increasing with time even though $\Delta \varepsilon_{\text {iso }}$ is still decreasing, suggesting that the isotropic phase is no longer the dominant phase. The increasing $\sigma_{\mathrm{dc}}$ with time in this time region should be mainly ascribed to the increasing $S$ of the nematic medium. Note that the conductivity of LCs is also anisotropic with generally $\sigma_{\|}>\sigma_{\perp}\left(\sigma_{\|}\right.$and $\sigma_{\perp}$ being the conductivity parallel and perpendicular to the director, respectively) [30].

The time dependence of $\tau_{\text {slow }}$ in time region II is particularly worth noting. Our previous study suggested that $\tau_{\text {slow }}$ has abnormal temperature dependence when LCMs go to the isotropic-in-nematic phase [13]. In this study, we notice that in time region II, where the sample is in the isotropic-in-nematic phase, $\tau_{\text {slow }}$ is decreasing with time, which means that this relaxation is being accelerated as time elapses. We believe this result originated from the same mechanism as that observed in the abnormal temperature dependence, that is, with fewer and fewer bulk isotropic 5CB molecules in the system, some 5CB molecules that were originally confined in the superstructure will be released and then transform to the nematic state, and the rotation of the remaining $5 \mathrm{CB}$ molecules in the superstructure will be accelerated because of the increasing free volume for each molecule to perform the rotation $[13,31]$. This result in turn confirms that a superstructure composed of water droplets and confined 5CB molecules is present in LCMs.

In time region III (after $700 \mathrm{~min}$ ), the fitting result shown in Fig. 5 indicates that there is no bulk isotropic phase in the effective measurement area. This is possibly because the isotropic 5CB molecules have totally diffused out of this area or because they are so few that their dielectric signal cannot be detected. No matter what the case is, the sample under measurement in this time region should be nearly free of the isotropic phase. Now that the isotropic phase takes $\sim 700 \mathrm{~min}$ to diffuse out of the effective measurement area $(2 \mathrm{~mm}$ in height); the self-diffusion coefficient of the isotropic phase can be roughly estimated to be $\sim 5 \times 10^{-11} \mathrm{~m}^{2} / \mathrm{s}$, which is a little smaller than the self-diffusion coefficient of bulk 5CB molecules. This is reasonable considering the heterogeneity of the system. This result in turn confirms that the upward diffusion of the isotropic phase in time region $\mathrm{I}$ is mainly induced by the hysteresis of the phase transition rather than the displacement of the isotropic phase.

It is very surprising that the slow process still exists and its dielectric strength even has a magnitude comparable to that of the librational mode of bulk nematic 5CB. Although it is thought that the nematic phase in LCM is nearly free of water droplets $[8,10]$, this result suggests that water droplets also exist in the nematic phase and can stably exist for
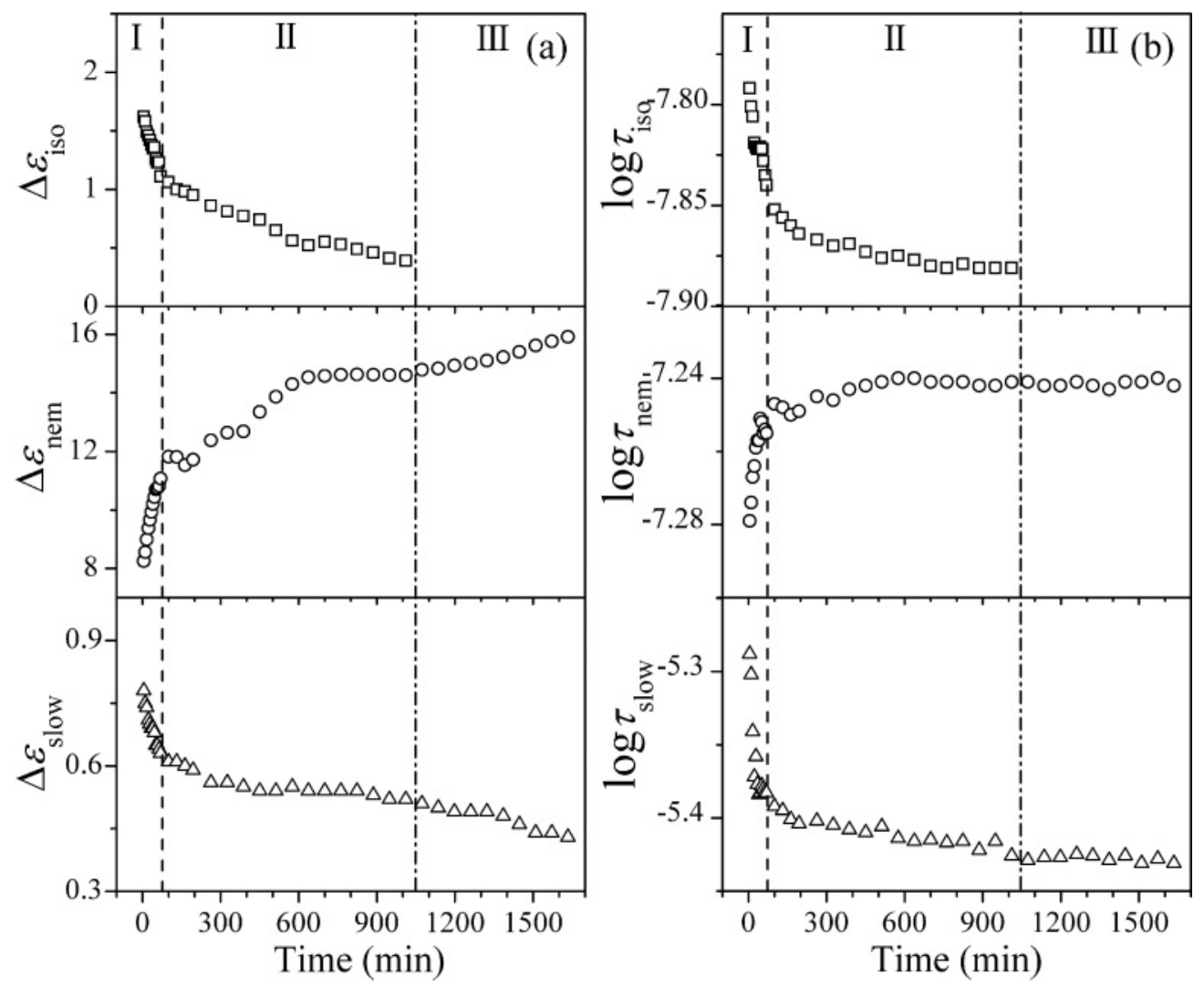

FIG. 7. Time dependence of (a) dielectric strength and (b) relaxation time of random reorientation of isotropic 5CB molecules (open square), rotation about short axis of nematic $5 \mathrm{CB}$ molecules (open circle), and slow process (open triangle) of the $0.15 \mathrm{LCM}$ at $20^{\circ} \mathrm{C}$. The broken lines indicate the boundaries of different time regions. 
a long period of time according to Fig. 5. Experimental observation in LC emulsions [1-3] and molecular dynamics simulation investigations [32] suggest that small particles can exist in nematic LCs, accompanied by defect structures such as hedgehog and Saturn ring. The water droplets in the present case may have analogous configurations, and their rotational dynamics may give rise to the slow process in this time region. However, even the rotation of "naked" water droplets will lead to a relaxation time much longer than the observed $\tau_{\text {slow }}$ [13]. Therefore, the mechanism of the slow process still goes to the dynamics of confined 5CB molecules, and the water droplets should still stay as the previously mentioned superstructures in time region III.

\section{Time-dependent dielectric behavior at $20^{\circ} \mathrm{C}$ (supercooled region)}

When the $0.15 \mathrm{LCM}$ is cooled to $20^{\circ} \mathrm{C}$, the sample is dominated by the supercooled nematic phase, but the isotropic phase still coexists although its concentration is very small $[8,10,13]$. The bulklike relaxations in this phase region have the same origin as that in the coexistence region. The slow process, however, suddenly shifts to a much lower frequency range; it is therefore believed to be attributed to the dynamics of water droplets instead of confined 5CB molecules in the superstructure [13]. The superstructure is possibly broken in this phase region because the paranematic fluctuation in this region is much stronger than that in the coexistence region. As a result, the water droplets may stay separately with configurations like hedgehog in the supercooled region. Figure 7 shows the time dependence of (a) $\Delta \varepsilon$ and (b) $\tau$ of the $0.15 \mathrm{LCM}$ at $20^{\circ} \mathrm{C}$, from which we can also clearly recognize three time regions.

In time region I (within $\sim 80 \mathrm{~min}$ ), all relaxation parameters have the most intensive time dependence. This should be a result of the hysteresis of NS phase transition and the upward diffusion of the isotropic phase (the former factor should also be the main one). In time region II (80-1150 min), the less intensive decrease in $\Delta \varepsilon_{\text {iso }}$ with time should be a result of only the upward diffusion of the isotropic phase. $\Delta \varepsilon_{\text {slow }}$ keeps almost constant in this time region although $\Delta \varepsilon_{\text {iso }}$ keeps decreasing, which implies that most water droplets exist in the supercooled nematic phase rather than the isotropic phase. Time region II is longer as compared with that in the case of $27.5^{\circ} \mathrm{C}$, indicating that the diffusion rate of the isotropic phase in the supercooled nematic phase is lower than in the nematic phase. In time region III, no bulk isotropic phase exists in the effective measurement area. It is worth noting that $\Delta \varepsilon_{\text {nem }}$ is still increasing while $\Delta \varepsilon_{\text {slow }}$ is decreasing with time. This result suggests that water droplets are also slowly diffusing out of the effective measurement area.

Figure 8 shows the time dependence of (a) the Cole-Cole parameter of the slow process and the short axis rotation of nematic 5CB molecules and (b) dc conductivity. Different from the case at $27.5^{\circ} \mathrm{C}, \sigma_{\mathrm{dc}}$ of the sample at $20^{\circ} \mathrm{C}$ has a monotonic dependence on time, which is because the continuous phase is always the supercooled nematic phase through the whole duration. Judging from the very small $\Delta \varepsilon_{\text {slow }}$, the concentration of water droplets in the supercooled nematic phase should be very low, and the formation of water droplet clusters as in the

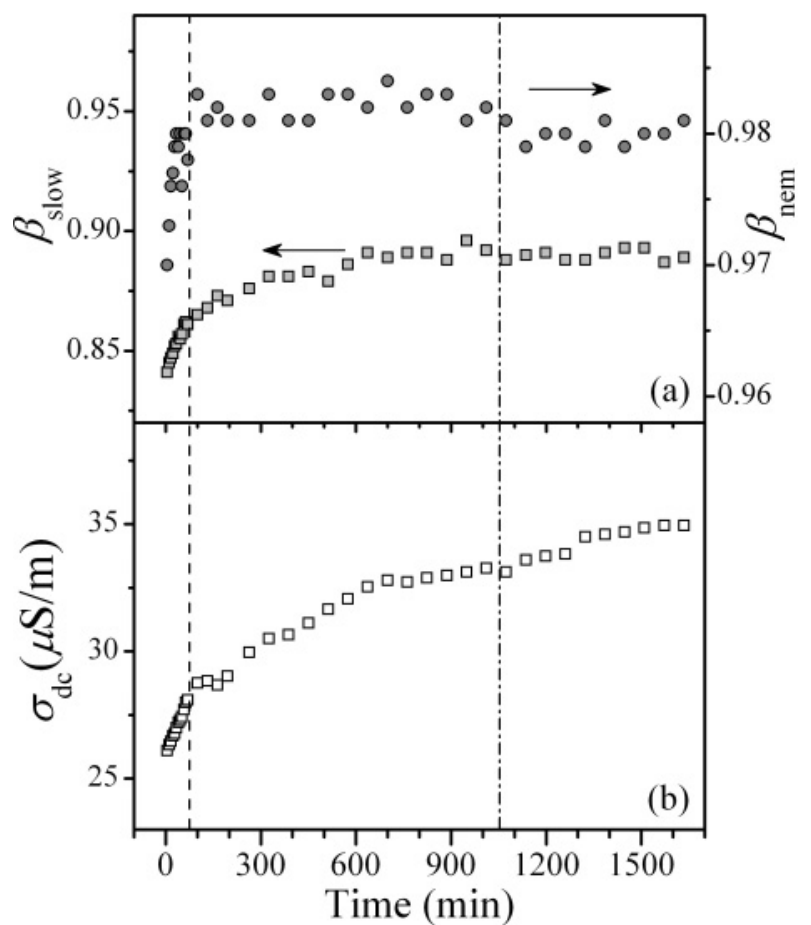

FIG. 8. Time dependence of the Cole-Cole parameter corresponding to (a) the slow process (filled square) and the relaxation due to short axis rotation of nematic 5CB molecules (filled circle) and (b) dc conductivity at $20^{\circ} \mathrm{C}$. The broken lines indicate the boundaries of different time regions.

case of $30^{\circ} \mathrm{C}$ is not possible. Therefore, $\sigma_{\mathrm{dc}}$ should be decided by the dynamics of the water droplets and the property of the dispersing medium, according to Eq. (3). In time regions II and III, we notice that $\beta_{\text {nem }}$ and $\tau_{\text {nem }}$ are almost constant, which means the ordering parameter of the supercooled nematic phase is barely changed after $80 \mathrm{~min}$; however, $\sigma_{\mathrm{dc}}$ is still increasing with time. Consequently, $\sigma_{\mathrm{dc}}$ should be mainly decided by the dynamics of the water droplets, now that the property of the dispersing medium does not change obviously. The increasing $\sigma_{\mathrm{dc}}$ with time suggests that the dynamics of the water droplets are increasing with time. On the other hand, a decrease in $\tau_{\text {slow }}$ with time is expected to be observed in these two time regions if the slow process is ascribed to the dynamics of water droplets. From Fig. 7(b) we can see $\tau_{\text {slow }}$ is indeed decreasing with time in these two time regions. This fact strongly confirms that the slow process in the supercooled region is attributed to the rotational diffusion of water droplets. Considering that the water droplets in the supercooled nematic phase may be accompanied by defect structures like hedgehog, we argue that these defect structures are also involved in the dynamics of the water droplets. These structures sometimes evolve with the variation of environment [32], which is probably responsible for the change of the dynamics of the water droplets.

\section{CONCLUSIONS}

The time-dependent dielectric behaviors of 5CB-DDABwater microemulsion in the isotropic, coexistence, and supercooled regions were monitored in real time by means of 
dielectric spectroscopy. It is found that the 5CB molecules in different states can be effectively characterized by dielectric spectroscopy even in such a kinetic process, and their dielectric behaviors in turn reflect the variation of the local phase composition with time.

In the isotropic region $\left(30^{\circ} \mathrm{C}\right)$, it is observed that water droplets start to diffuse to the upper part of the container at $\sim 300 \mathrm{~min}$, but the property of bulk isotropic 5CB is barely changed with time. It is confirmed that the slow process is ascribed to the retarded rotation of the $5 \mathrm{CB}$ molecules on the surface of the water droplets.

Three time regions labeled by different dielectric behavior are observed for the sample both in the coexistence region $\left(27.5^{\circ} \mathrm{C}\right)$ and in the supercooled region $\left(20^{\circ} \mathrm{C}\right)$. The remarkable change of the dielectric behavior in the first time region of both cases suggests that the IN and NS transitions present a hysteresis effect. In the coexistence region, a microscopic phase evolution from a nematic-in-isotropic via a bicontinuous to an isotropic-in-nematic phase is observed, which is similar to the temperature-induced phase evolution observed in our previous study. The dielectric behavior of the slow process during this evolution confirms that this process is ascribed to the dynamics of 5CB molecules confined in the superstructure. The slow process is still observed even after the bulk isotropic phase has been totally excluded out of the effective measurement area, which suggests that the superstructure can stably exist in the nematic phase for a long period of time. In the supercooled region, the slow process is also observed after the bulk isotropic phase has been totally excluded, indicating that the water droplets can also exist in the supercooled nematic phase. It is found that the time dependences of $\tau_{\text {slow }}$ and $\sigma_{\mathrm{dc}}$ are interrelated, which confirms that the slow process is ascribed to the rotational diffusion of the water droplets.

In conclusion, this study further suggests that a superstructure composed of water droplets and confined 5CB molecules exists in the 5CB-DDAB-water microemulsion after the IN phase transition. This study also suggests that water droplets can stably exist in the liquid crystalline phase, although this phase is generally thought to be free of them. Because the phase behavior of such system is vulnerable to many factors such as the geometry of the sample cell, more dielectric spectroscopy investigation under different condition is highly desirable.

\section{ACKNOWLEDGMENTS}

This work is partly supported by KAKENHI (Grant No. 19340116), Grant-in-Aid for Scientific Research (B), from the Ministry of Education, Culture, Sports, Science, and Technology (MEXT) of Japan.
[1] P. Poulin, H. Stark, T. C. Lubensky, and D. A. Weitz, Science 275, 1770 (1997).

[2] P. Poulin and D. A. Weitz, Phys. Rev. E 57, 626 (1998).

[3] P. Poulin, Curr. Opin. Colloid Interface Sci. 4, 66 (1999).

[4] E. M. Terentjev, Europhys. Lett. 32, 607 (1995).

[5] E. M. Terentjev, in Modern Aspects of Colloidal Dispersions, edited by R. H. Ottewill and A. R. Rennie (Kluwer Academic, London, 1998), pp. 257-268.

[6] J. Yamamoto and H. Tanaka, Nature (London) 405, 321 (2001).

[7] T. Bellini, M. Caggioni, N. A. Clark, F. Mantegazza, A. Maritan, and A. Pelizzola, Phys. Rev. Lett. 91, 085704 (2003).

[8] M. Caggioni, A. Giacometti, T. Bellini, N. A. Clark, F. Mantegazza, and A. Maritan, J. Chem. Phys. 122, 214721 (2005).

[9] Z. Kutnjak, G. Cordoyiannis, G. Nounesis, A. Lebar, B. Zalar, and S. Žumer, J. Chem. Phys. 122, 224709 (2005).

[10] A. Lebar, Z. Kutnjak, H. Tanaka, B. Zalar, and S. Žumer, Phys. Rev. E 78, 031707 (2008).

[11] G. Toquer, G. Porte, M. Nobili, J. Appell, and C. Blanc, Langmuir 23, 4081 (2007).

[12] M. Adams, Z. Dogic, S. L. Keller, and S. Fraden, Nature (London) 393, 349 (1998).

[13] Z. Chen and R. Nozaki, J. Chem. Phys. 134, 034505 (2011).

[14] F. Kremer and A. Schonhals, Broadband Dielectric Spectroscopy (Springer, Berlin, 2003).

[15] A. Schönhals, A. Fritz, and K. Pfeiffer, Macromol. Chem. Phys. 202, 3228 (2001).

[16] C. Hakmé, I. Stevenson, A. Maazouz, P. Cassagnau, G. Boiteux, and G. Seytre, J. Non-Cryst. Solids 353, 4362 (2007).
[17] A. Minoguchi and R. Nozaki, J. Non-Cryst. Solids 307-310, 246 (2002).

[18] A. R. Brás, M. T. Viciosa, Y. Wang, M. Dionísio, and J. F. Mano, Macromolecules 39, 6513 (2006).

[19] S. Napolitano and M. Wübbenhorst, J. Non-Cryst. Solids 353, 4357 (2007).

[20] B. D. Fitz and J. Mijovic, J. Phys. Chem. B 104, 12215 (2000).

[21] S. Havriliak and S. Negami, Polymer, 8, 161 (1967).

[22] K. S. Cole and R. H. Cole, J. Chem. Phys. 9, 341 (1941).

[23] H. F. Eicke, M. Bercovec, and B. Das-Gupta, J. Phys. Chem. 93, 314 (1989).

[24] Y. Feldman, N. Kozlovich, I. Nir, and N. Garti, Phys. Rev. E 51, 478 (1995).

[25] A. Dukhin and P. Goetz, Colloids Surf., A 253, 51 (2005).

[26] J. G. Kirkwood, J. Chem. Phys. 7, 911 (1939).

[27] H. Fröhlich, Theory of Dielectrics, 2nd ed. (Oxford University Press, Oxford, 1958).

[28] M. Vilfan, T. Apih, A. Gregorovič, B. Zalar, G. Lahajnar, S. Žumer, G. Hinze, R. Bohmer, and G. Althoff, Magn. Reson. Imaging 19, 433 (2001).

[29] V. V. Daniel, Dielectric Relaxation (Academic, New York, 1967).

[30] L. M. Blinov and V. G. Chigrinov, Electrooptic Effects in Liquid Crystal Materials (Springer, New York, 1994).

[31] Y. E. Ryabov, A. Puzenko, and Y. Feldman, Phys. Rev. B 69, 014204 (2004).

[32] J. Fukuda, J. Phys. Soc. Jpn. 78, 041003 (2009), and references therein. 